\title{
A proof of hypoellipticity for Kohn's operator via FBI
}

\section{Gregorio Chinni}

\begin{abstract}
A new proof of both analytic and $C^{\infty}$ hypoellipticity of Kohn's operator is given using FBI techniques introduced by J. Sjöstrand. The same proof allows us to obtain both kind of hypoellipticity at the same time.
\end{abstract}

\section{Introduction}

In 2005, J. J. Kohn published a seminal paper [6], mainly concerned with the problem of $C^{\infty}$-hypoellipticity for sums of squares of complex vector fields.

It turns out that, in contrast with the case of real vector fields satisfying Hörmander's bracket condition, a sum of squares of complex vector fields can be hypoelliptic and, at the same time, lose an arbitrary number of derivatives. As a consequence, the primary tool (the a priori estimate) used to prove hypoellipticity can be rather difficult to obtain. As a matter of fact, J. J. Kohn in [6] produced an example of a sum of squares of complex vector fields with real analytic coefficients having a symplectic characteristic manifold that is $C^{\infty}$ - hypoelliptic and, as Derridj and Tartakoff prove in the appendix to [6], is also analytic hypoelliptic.

We recall that a sum of squares of complex vector fields is related to a sum of squares of real vector fields with special lower order terms. In this situation, in a transversally non-degenerate case, Treves [14] and Kwon [7, 8] have proved both $C^{\infty}$ and real analytic hypoellipticity.

The purpose of the present paper is to give an alternate proof of both Kohn's result and that of Derridj and Tartakoff on Kohn's model operator,

2000 Mathematics Subject Classification: 35H10, 35A20, 35B65.

Keywords: Analytic hypoellipticity, FBI transform, sum of squares of complex vector fields. 
or rather that studied in [2]. We use F.B.I. transform techniques. The advantage of such an approach is that the same proof works in both the $C^{\infty}$ and $C^{\omega}$ categories at the same time. This is due to the fact that, roughly speaking, the F.B.I. transform has a built in localization that is well adapted to the analytic category. This results in a characterization of both the $C^{\infty}$ wave front set, $W F$, and the analytic wave front set, $W F_{a}$, with a uniform decay rate (near a certain point) of the F.B.I. transform of the distribution in examination (see, e.g., [10]). The technique we use to derive an a priori estimate on the F.B.I. side is inspired by the work of J. Sjöstrand [11]. A similar technique, not dealing with the spectral degeneracy involved in Kohn's operator, has permitted J. Sjöstrand to give an F.B.I. proof of the analytic hypoellipticity results of Treves [15] and Tartakoff $[12,13]$.

Let us now state our theorem. Let $q$ be an even positive integer and $k$ a positive integer. Define

$$
L=D_{x}+i x^{q-1} D_{t},
$$

where $D_{x}=i^{-1} \partial_{x}, x \in \mathbb{R}$. The Kohn's operator is defined as

$$
P\left(x, D_{x}, D_{t}\right)=L L^{*}+L^{*} x^{2 k} L .
$$

Then we have the

Theorem 1.1. Kohn's operator (1.1) is both $C^{\infty}$ and $C^{\omega}$ hypoelliptic.

We point out that the operator $P$ is considered only when $q$ is even and positive. As a matter of fact the case when $q$ is an odd integer is much easier since then $P$ is both $C^{\infty}$ and $C^{\omega}$ hypoelliptic with a loss of one derivative. This may be explained by remarking that the kernel of $L_{\tau}^{*}$ is empty in the distribution category, where $L_{\tau}^{*}=D_{x}-i x^{q-1} \tau$, since $L_{\tau}^{*} e^{-x^{q} \tau / q}=0$.

\section{Construction of the left parametrix}

Let $\lambda$ be a positive large parameter. Consider the operator $\lambda^{-2} P$ :

$$
\left(\tilde{D}_{x}+i x^{q-1} \tilde{D}_{t}\right)\left(\tilde{D}_{x}-i x^{q-1} \tilde{D}_{t}\right)+\left(\tilde{D}_{x}-i x^{q-1} \tilde{D}_{t}\right) x^{2 k}\left(\tilde{D}_{x}+i x^{q-1} \tilde{D}_{t}\right),
$$

where we used the notation $\tilde{D}_{x}=\frac{1}{\lambda \sqrt{-1}} \frac{\partial}{\partial x}$ and $\tilde{D}_{t}=\frac{1}{\lambda \sqrt{-1}} \frac{\partial}{\partial t}$.

The symbol associated with (1.1) via $\lambda$-Fourier transform

$$
\begin{aligned}
& \hat{u}(\xi, \tau)=\int e^{-i \lambda(x \xi+t \tau)} u(x, t) d x d t, \\
& u(x, t)=\left(\frac{\lambda}{2 \pi}\right)^{2} \int e^{i \lambda(x \xi+t \tau)} \hat{u}(\xi, \tau) d \xi d \tau,
\end{aligned}
$$


is

$$
\begin{aligned}
\left(1+x^{2 k}\right)\left(\xi^{2}+\right. & \left.x^{2(q-1)} \tau^{2}\right)+ \\
& +\frac{1}{\lambda}\left(\left(x^{2 k}-1\right)(q-1) x^{q-2} \tau-2 i k x^{2 k-1}\left(\xi+i x^{q-1} \tau\right)\right) .
\end{aligned}
$$

Its characteristic set is

$$
\Sigma=\left\{(x, t, \xi, \tau) \in \mathbb{R}^{4} \backslash\{0\}: \xi=0=x \text { with } \tau \neq 0\right\} ;
$$

we put $\Sigma=\Sigma^{+} \cup \Sigma^{-}$where $\Sigma^{ \pm}=\{\rho \in \Sigma: \tau \gtrless 0\}$. Since the theorem we want to prove is microlocal in essence, we shall argue in a neighborhood of the point $\rho_{0}=(0,0,0,1) \in \Sigma^{+}$.

Let us consider the $\lambda$-Fourier transform of the operator $P$ with respect to the variable $t$

$$
\begin{aligned}
\tilde{D}_{x}^{2}+ & x^{2(q-1)} \tau^{2}-\frac{q-1}{\lambda} x^{q-2} \tau+ \\
& +x^{2 k}\left(\tilde{D}_{x}^{2}+x^{2(q-1)} \tau^{2}+\frac{q-1}{\lambda} x^{q-2} \tau\right)-\frac{2 i k}{\lambda} x^{2 k-1}\left(\tilde{D}_{x}+i x^{q-1} \tau\right)
\end{aligned}
$$

It is useful adopt the notation:

1. $P_{0}=\tilde{D}_{x}^{2}+x^{2(q-1)} \tau^{2}-\frac{q-1}{\lambda} x^{q-2} \tau$,

2. $P_{k}=x^{2 k}\left(\tilde{D}_{x}^{2}+x^{2(q-1)} \tau^{2}+\frac{q-1}{\lambda} x^{q-2} \tau\right)-\frac{2 i k}{\lambda} x^{2 k-1}\left(\tilde{D}_{x}+i x^{q-1} \tau\right)$.

We point out that the above subdivision of $P$ reflects a homogeneity property. More precisely let us consider the dilation $x \mapsto \lambda^{-1 / q} x, t \mapsto t$ as well as its canonical action on the covariables $\xi \mapsto \lambda^{1 / q} \xi, \tau \mapsto \tau$. Then if $p_{0}, p_{k}$ denote the symbol of $P_{0}$ and $P_{k}$ w.r.t. the usual Fourier transform, we have

$$
\begin{aligned}
& p_{0}\left(\lambda^{-1 / q} x, \lambda^{1 / q} \xi / \lambda, \tau\right)=\lambda^{-2+\frac{2}{q}} p_{0}(x, \xi, \tau) \text { and } \\
& p_{k}\left(\lambda^{-1 / q} x, \lambda^{1 / q} \xi / \lambda, \tau\right)=\lambda^{-2+\frac{2}{q}-\frac{2 k}{q}} p_{k}(x, \xi, \tau) .
\end{aligned}
$$

We call this homogeneity property "global homogeneity".

To construct an approximate parametrix for $P_{0}+P_{k}$, which will be sufficient for our purpose, we shall use an algebraic technique following Sjöstrand [9]. We use the same framework as Boutet de Monvel [1]. In the latter paper classes of symbols are studied via the distance function to a symplectic characteristic manifold. We use basically the same classes, replacing quadratic distance with an adapted anisotropic function, keeping into account the higher vanishing order w.r.t. the $x$ variable of $p(x, \xi, \tau)$. We set

$$
m(x, \xi, \lambda)=\left(d_{\Sigma}^{2}+\lambda^{-\frac{2(q-1)}{q}}\right)^{\frac{1}{2}} \text { where } d_{\Sigma}^{2}=|\xi|^{2}+|x|^{2(q-1)}
$$


and we say that a $C^{\infty}$ function $a$ belongs to $S_{q}^{m, k}\left(\mathbb{R}_{x, t}^{2} \times \mathbb{R}_{\xi, \tau}^{2}, \Sigma\right)$, or briefly $S_{q}^{m, k}$, if for any positive integers $\gamma, \mu, \alpha$ and $\beta$ we have

$$
\left|\partial_{\tau}^{\gamma} \partial_{t}^{\mu} \partial_{\xi}^{\beta} \partial_{x}^{\alpha} a(x, t, \xi, \tau)\right| \lesssim \lambda^{m-\beta-\gamma} m(x, \xi, \lambda)^{k-\beta-\frac{\alpha}{q-1}}
$$

for $\lambda \geq 1$ and $(x, t, \xi, \tau)$ in a neighborhood of $(0,0,0,1) \in \Sigma^{+}$. In the same way we define the symbol class $\mathscr{H}_{q}^{m}=\cap_{j=0}^{\infty} S_{q}^{m-j, k-j q /(q-1)}$ (see [1] for more details.) We denote by $O P \mathscr{H}_{q}^{m}=\cap_{j=0}^{\infty} O P S_{q}^{m-j, k-j q /(q-1)}$ the set of pseudodifferential operator corresponding to $\mathscr{H}_{q}^{m}$.

We recall the inclusion relation: $S_{q}^{m, k} \subseteq S_{q}^{m^{\prime}, k^{\prime}}$ if and only if $m \leq m^{\prime}$ and $m-k(q-1) / q \leq m^{\prime}-k^{\prime}(q-1) / q$.

We have that

$$
P_{0}\left(x, \tilde{D}_{x}, \tilde{D}_{t}\right) \in O P S_{q}^{0,2} \quad \text { and } \quad P_{k}\left(x, \tilde{D}_{x}, \tilde{D}_{t}\right) \in O P S_{q}^{0,2+\frac{2 k}{q-1}} .
$$

Let $\Sigma_{1}=\Pi_{x}(\Sigma)$ be the projection of characteristic set $\Sigma$ on $\mathbb{R}_{x}$. We define the space $\mathcal{H}_{q}^{m+1 / 2 q}\left(\mathbb{R}_{x, t}^{2} \times \mathbb{R}_{\tau}, \Sigma_{1}\right)$, or shortly $\mathcal{H}_{q}^{m+1 / 2 q}$, as the space of all smooth functions belonging to $\cap_{j=0}^{\infty} S_{q}^{m-j+1 / 2 q,-j q /(q-1)}\left(\mathbb{R}_{x, t}^{2} \times \mathbb{R}_{\tau}, \Sigma_{1}\right)$, where $S_{q}^{m, k}\left(\mathbb{R}_{x, t}^{2} \times \mathbb{R}_{\tau}, \Sigma_{1}\right)$ denotes the set of all smooth functions such that

$$
\left|\partial_{\tau}^{\gamma} \partial_{t}^{\mu} \partial_{x}^{\alpha} a(x, t, \tau, \lambda)\right| \lesssim \lambda^{m-\gamma}\left(|x|^{2(q-1)}+\lambda^{-\frac{2(q-1)}{q}}\right)^{\frac{k}{2}-\frac{\alpha}{2(q-1)}} .
$$

The action of a symbol $a$ in $\mathcal{H}_{q}^{m+1 / 2 q}$ as a map $a\left(x, t, \tilde{D}_{t}\right): C_{0}^{\infty}\left(\mathbb{R}_{t}\right) \rightarrow$ $C^{\infty}\left(\mathbb{R}_{x, t}^{2}\right)$ is defined by

$$
\left(a\left(x, t, \tilde{D}_{t}, \lambda\right) u\right)(x, t)=\frac{\lambda}{2 \pi} \int e^{i \lambda\left(t-t^{\prime}\right) \tau} a(x, t, \tau) u\left(t^{\prime}\right) d t^{\prime} d \tau .
$$

Such an operator, modulo a regularizing operator (w.r.t. the $t$-variable) is called an Hermite operator of degree $m$ and we denote by $O P \mathcal{H}_{q}^{m}$ the corresponding operator class.

Let $a \in \mathcal{H}_{q}^{m+1 / 2 q}$, we define the adjoint of the Hermite operator $a$ as the $\operatorname{map} a^{*}\left(x, t, \tilde{D}_{t}, \lambda\right): C_{0}^{\infty}\left(\mathbb{R}_{x, t}^{2}\right) \rightarrow C^{\infty}\left(\mathbb{R}_{t}\right)$ defined by

$$
\left(a^{*}\left(x, t, \tilde{D}_{t}, \lambda\right) u\right)(t)=\frac{\lambda}{2 \pi} \iint e^{i \lambda\left(t-t^{\prime}\right) \tau} \overline{a(x, t, \tau)} u\left(x, t^{\prime}\right) d x d t^{\prime} d \tau
$$

We denote by $O P \mathcal{H}_{q}^{*} m$ the related space of operators.

$P_{0}\left(x, \tilde{D}_{x}, \tau\right)$ is a self-adjoint operator on $\mathscr{S}\left(\mathbb{R}_{x}\right)$; moreover, since $q$ is even, it is not injective and actually it has an one dimensional kernel. For more details on this subject see [3]. 
Let $e_{0, \tau}(x, \lambda)$ be the $L^{2}$-normalized null eigenfunction of $P_{0},\left\|e_{0, \tau}\right\|_{L^{2}}=1$,

$$
e_{0, \tau}(x, \lambda)=\sqrt{c_{q}} \lambda^{\frac{1}{2 q}} \tau^{\frac{1}{2 q}} e^{-\frac{\lambda \tau x^{q}}{q}}
$$

where $c_{q}=\frac{2}{q} \sqrt[q]{\frac{q}{2}} \Gamma\left(\frac{1}{q}\right)$. We remark that $e_{0, \tau}$ belongs to the space $\mathcal{H}_{q}^{1 / 2 q}$ and the operator

$$
e_{0}\left(x, \tilde{D}_{t}, \lambda\right) f(x, t)=\frac{\lambda}{2 \pi} \int e^{i \lambda t \tau} e_{0, \tau}(x, \lambda) \hat{f}(\tau) d \tau
$$

is an Hermite operator of degree $0, e_{0}\left(x, \tilde{D}_{t}, \lambda\right) \in O P \mathcal{H}_{q}^{0}$. We recall that, by the results of [4] and [5], we have

$$
\left|\hat{e}_{0, \tau}(\xi, \lambda)\right| \leq c \lambda^{-\frac{1}{2 q}} \tau^{-\frac{1}{2 q}} e^{-\varepsilon \lambda \tau^{-\frac{1}{q-1}} \xi^{\frac{q}{q-1}}}
$$

where $c$ and $\varepsilon$ are suitable positive constants.

We define the operators

$$
E: L^{2}\left(\mathbb{R}_{\tau}\right) \longrightarrow L^{2}\left(\mathbb{R}_{x, \tau}^{2}\right), \quad E(f(\tau))=e_{0, \tau}(x, \lambda) f(\tau)
$$

and

$$
E^{*}: L^{2}\left(\mathbb{R}_{x, \tau}^{2}\right) \longrightarrow L^{2}\left(\mathbb{R}_{\tau}\right), \quad E^{*}(u(x, \tau))=\int e_{0, \tau}(x, \lambda) u(x, \tau) d x .
$$

In order to obtain an approximate parametrix of the operator $P$ we begin by constructing an inverse of the matrix operator

$$
\mathfrak{P}=\left(\begin{array}{cc}
\left(P_{0}+P_{k}\right)\left(x, \tilde{D}_{x}, \tau\right) & E \\
E^{*} & 0
\end{array}\right) .
$$

The operator $P_{0}\left(x, \tilde{D}_{x}, \tau\right)$ on $\left\langle e_{0, \tau}\right\rangle^{\perp}$, the $L^{2}$-orthogonal complement of the one dimensional spaces generated by $e_{0, \tau}$, is injective and admits an inverse. We have that the matrix

$$
\left(\begin{array}{cc}
P_{0}\left(x, \tilde{D}_{x}, \tau\right) & E \\
E^{*} & 0
\end{array}\right)
$$

is a bijection on $\mathscr{S}\left(\mathbb{R}_{x, \tau}^{2}\right) \times L^{2}\left(\mathbb{R}_{\tau}\right)$ and it has an inverse:

$$
\left(\begin{array}{cc}
F_{0}\left(x, \tilde{D}_{x}, \tau\right) & E \\
E^{*} & 0
\end{array}\right)
$$


where $F_{0}\left(x, \tilde{D}_{x}, \tau\right)$ is a parametrix of $P_{0}\left(x, \tilde{D}_{x}, \tau\right)$ restricted to the range of $1-E E^{*}$ :

$$
P_{0} \# F_{0}=1-E E^{*} .
$$

Here \# denotes the Weyl composition. The operator $F_{0}\left(x, \tilde{D}_{x}, \tilde{D}_{t}\right)$ belongs to $O P S_{q}^{0,-2}$. We remark that $1-E E^{*}$ is the projection on $\left\langle e_{0, \tau}\right\rangle^{\perp}$.

We have

$$
\begin{gathered}
\left(\begin{array}{cc}
F_{0}\left(x, \tilde{D}_{x}, \tau\right) & E \\
E^{*} & 0
\end{array}\right)\left(\begin{array}{cc}
\left(P_{0}+P_{k}\right)\left(x, \tilde{D}_{x}, \tau\right) & E \\
E^{*} & 0
\end{array}\right)= \\
=\left(\begin{array}{cc}
1+F_{0} P_{k}\left(x, \tilde{D}_{x}, \tau\right) & 0 \\
E^{*} P_{k} & 1
\end{array}\right)
\end{gathered}
$$

Here $F_{0} P_{k}\left(x, \tilde{D}_{x}, \tilde{D}_{t}\right) \in O P S_{q}^{0, \frac{2 k}{q-1}}$; a direct computation shows that $P_{k} e_{0, \tau}$ is equal to $\lambda^{-1} 2(2 k+q-1) \tau x^{2 k+q-2} e_{0, \tau}$, then we have that $E^{*} P_{k}$ belongs to $O P \mathcal{H}_{q}^{*-\frac{2 k}{q}-\frac{2(q-1)}{q}}$. We recall that if $A \in O P S_{q}^{m, k}$ and $H \in O P \mathscr{H}_{q}^{m^{\prime}}$ then $A H \in O P \mathscr{H}_{q}^{m+m^{\prime}-k(q-1) / q}$. We remark that the operator $1+F_{0} P_{k}$ satisfies the second condition of the Proposition 6.1 of [1] so it admits a left parametrix. $\left.{ }^{1}\right)$

The inverse of the matrix in the right hand side of (2.8) is

$$
\left(\begin{array}{cc}
\left(1+F_{0} P_{k}\right)^{-1} & 0 \\
-E^{*} P_{k}\left(1+F_{0} P_{k}\right)^{-1} & 1
\end{array}\right) .
$$

Formally we have $\left(1+F_{0} P_{k}\right)^{-1}=1-\sum_{j \geq 0}(-1)^{j}\left(F_{0} P_{k}\right)^{j+1}$, for every $j$ we have $\sigma\left(F_{0} P_{k}\right)^{j+1} \in S_{q}^{0, \frac{2 k}{q-1}+j \frac{2 k}{q-1}}$. By the Proposition 1.11, of [1], exists a symbol $r \in S_{q}^{0, \frac{2 k}{q-1}}$ such that for all $N, r-\sum_{j \leq N}(-1)^{j} \sigma\left(F_{0} P_{k}\right)^{j+1}$ belongs to $S_{q}^{0, \frac{2 k}{q-1}+\frac{N}{q-1}}$. Let $R \sim \sum(-1)^{j+1}\left(F_{0} P_{k}\right)^{j}$ modulo $O P S_{q}^{0, \infty}$. We can write the inverse of the matrix $\mathfrak{P}$

$$
\begin{aligned}
\mathfrak{Q}=\left(\begin{array}{cc}
\left(1+F_{0} P_{k}\right)^{-1} & 0 \\
-E^{*} P_{k}\left(1+F_{0} P_{k}\right)^{-1} & 1
\end{array}\right)\left(\begin{array}{cc}
F_{0} & E \\
E^{*} & 0
\end{array}\right) \\
=\left(\begin{array}{cc}
\left(1+F_{0} P_{k}\right)^{-1} F_{0} & \left(1+F_{0} P_{k}\right)^{-1} E \\
E^{*}-E^{*} P_{k}\left(1+F_{0} P_{k}\right)^{-1} F_{0} & -E^{*} P_{k}\left(1+F_{0} P_{k}\right)^{-1} E
\end{array}\right)
\end{aligned}
$$

\footnotetext{
${ }^{1}$ Actually if an operator $A \in O P S_{q}^{m, k}$ satisfies condition (ii) of the Proposition 6.1 then the operator $A+B$, with $B \in O P S_{q}^{m, k+k^{\prime}}$ and $k^{\prime} \geq 1$, satisfies the same condition.
} 
We have that $\mathfrak{Q P} \sim I d$, this relation gives

$$
\left\{\begin{array}{l}
\left(\left(1+F_{0} P_{k}\right)^{-1} F_{0}\right)\left(P_{0}+P_{k}\right)+\left(1+F_{0} P_{k}\right)^{-1} E E^{*}=1 ; \\
\left(E^{*}-E^{*} P_{k}\left(1+F_{0} P_{k}\right)^{-1} F_{0}\right)\left(P_{0}+P_{k}\right)-E^{*} P_{k}\left(1+F_{0} P_{k}\right)^{-1} E E^{*}=0 .
\end{array}\right.
$$

Using the second equation in above system we want to show that $E^{*}=$ $S\left(P_{0}+P_{k}\right)+O P \mathcal{H}_{q}^{*-\frac{2 k}{q} N-\frac{1}{q}}$ for a suitable operator $S$. For this purpose we give an expression of $E^{*} P_{k}\left(1+F_{0} P_{k}\right)^{-1} E$ (as an operator in $\lambda$ and $\tau$.) We have

$$
P_{k}\left(1+F_{0} P_{k}\right)^{-1} E=P_{k} E+\sum_{j \geq 0}(-1)^{j+1} P_{k}\left(F_{0} P_{k}\right)^{j+1} E
$$

For any $j$, the symbol associated to the operator $P_{k}\left(F_{0} P_{k}\right)^{j+1} E$ belongs to the class $S_{q}^{-\frac{4 k}{q}-\frac{2(q-1)}{q}-\frac{j}{q},-\frac{j}{q-1}}$. By $[1$, Proposition 1.11], there exists an $r_{1} \in S_{q}^{-\frac{4 k}{q}-\frac{2(q-1)}{q}, 0}$ such that, for all $N$

$$
r_{1}-\sum_{j \geq 0}^{N} \sigma\left(P_{k}\left(F_{0} P_{k}\right)^{j+1} E\right) \in S_{q}^{-\frac{4 k}{q}-\frac{2(q-1)}{q}-\frac{N}{q},-\frac{N}{q-1}}
$$

$\left(r_{1}\right.$ is defined modulo an element in $\mathscr{H}_{q}^{-\frac{4 k}{q}-\frac{2(q-1)}{q}}$.)

We have that

$$
\begin{aligned}
& E^{*} P_{k}\left(1+F_{0} P_{k}\right)^{-1} E=E^{*} P_{k} E+ \\
& \quad+\sum_{j=0}^{N-1}(-1)^{j+1} P_{k}\left(F_{0} P_{k}\right)^{j+1} E+\sum_{l \geq 0}(-1)^{l+N} P_{k}\left(F_{0} P_{k}\right)^{l+N} E .
\end{aligned}
$$

$E^{*} P_{k} E$ is the leading symbol, $\sigma\left(E^{*} P_{k} E\right) \sim \tau^{-\frac{2 k}{q}+\frac{2}{q}} \lambda^{-\frac{2 k}{q}-\frac{2(q-1)}{q}}$.

We point out that the number $\frac{2 k}{q}+\frac{2(q-1)}{q}$, appearing as the exponent of $\lambda$, is the loss of derivatives of $P$.

Furthermore

$$
E^{*} \sum_{j \geq 0}^{N-1}(-1)^{j+1} P_{k}\left(F_{0} P_{k}\right)^{j+1} E
$$

is a symbol in $\tau$ and $\lambda$ of order $-\frac{4 k}{q}-\frac{2(q-1)}{q}$ and

$$
E^{*} \sum_{l \geq 0} P_{k}\left(F_{0} P_{k}\right)^{l+N} E
$$

is a symbol in $\tau$ and $\lambda$ of order $-\frac{2 k}{q}(N+1)-\frac{2(q-1)}{q}$. 
Then we have

$$
\sigma\left(E^{*} P_{k}\left(1+F_{0} P_{k}\right)^{-1} E\right) \sim A+B
$$

here

$$
A=\lambda^{-\frac{2 k}{q}-\frac{2(q-1)}{q}} \tau^{-\frac{2 k}{q}+\frac{2}{q}}\left(1-\sum_{j=1}^{N-1} c_{j} \tau^{-\frac{2 k}{q} j} \lambda^{-\frac{2 k}{q} j}\right)
$$

and

$$
B=c_{N} \tau^{-\frac{2 k}{q}(N+1)+\frac{2}{q}} \lambda^{-\frac{2 k}{q}(N+1)-\frac{2(q-1)}{q}}(1+R(t, \tau, \lambda))
$$

where $R$ is a symbol of order $-\frac{2 k}{q}$. Using the second equation in (2.10) we deduce that

$$
E^{*}=A^{-1} B E^{*}+A^{-1}\left(E^{*}-E^{*} P_{k}\left(1-F_{0} P_{k}\right)^{-1} F_{0}\right)\left(P_{0}+P_{k}\right)
$$

or more explicitly

$$
\begin{aligned}
E^{*}=\frac{\tau^{\frac{2 k}{q}-\frac{2}{q}} \lambda^{\frac{2 k}{q}+\frac{2(q-1)}{q}}}{\left(1-\sum_{j=1}^{N-1} c_{j} \tau^{-\frac{2 k}{q} j} \lambda^{-\frac{2 k}{q} j}\right)} & \left(E^{*}-E^{*} P_{k}\left(1-F_{0} P_{k}\right)^{-1} F_{0}\right)\left(P_{0}+P_{k}\right) \\
& +c_{N} \tau^{-\frac{2 k}{q} N} \lambda^{-\frac{2 k}{q} N} E^{*} \quad \bmod \left(\mathcal{H}_{q}^{*-\frac{2 k}{q} N-\frac{1}{q}}\right) .
\end{aligned}
$$

Hence

$$
\begin{array}{r}
E^{*}=\tau^{\frac{2 k}{q}-\frac{2}{q}} \lambda^{\frac{2 k}{q}+\frac{2(q-1)}{q}} \sum_{s<N+1+\frac{q-1}{k}} \tilde{c}_{s}(\tau \lambda)^{-\frac{2 k}{q} s}\left(E^{*}-E^{*} P_{k}\left(1-F_{0} P_{k}\right)^{-1} F_{0}\right)\left(P_{0}+P_{k}\right) \\
+c_{N} \tau^{-\frac{2 k}{q} N} \lambda^{-\frac{2 k}{q} N} E^{*} \quad \bmod \left(\mathcal{H}_{q}^{*-\frac{2 k}{q} N-\frac{1}{q}}\right) .
\end{array}
$$

where $\tilde{c}_{s}$ are real constants. Using the above representation of $E^{*}$ and the first equation in (2.10) we get

$$
\begin{aligned}
& \left(\tau^{\frac{2 k}{q}-\frac{2}{q}} \lambda^{\frac{2 k}{q}+\frac{2(q-1)}{q}} \sum \tilde{c}_{s}(\tau \lambda)^{-\frac{2 k}{q} s} E E^{*}\left(1+P_{k}\left(1+F_{0} P_{k}\right)^{-1} F_{0}\right)+\right. \\
& \left.\quad+\left(1+F_{0} P_{k}\right)^{-1} F_{0}\right)\left(P_{0}+P_{k}\right)=1-\tau^{-\frac{2 k}{q} N} \lambda^{-\frac{2 k}{q} N} E E^{*} \bmod \left(\mathscr{H}_{q}^{-\frac{2 k}{q} N-\frac{1}{q}}\right) .
\end{aligned}
$$

The term

$$
\tau^{-\frac{2 k}{q} N} \lambda^{-\frac{2 k}{q} N} E E^{*} \in O P S_{q}^{-\frac{2 k}{q} N, 0} \subset O P S_{q}^{-\frac{1}{q},-\frac{1}{q-1}} .
$$

We do not give a direct construction of the parametrix but, from the condition (i) of the Proposition 6.1 ([1]), it is easy to see that the operator $\lambda^{-2} P$ admits a left parametrix. For future purposes we state here what has been done until now. 
Proposition 2.1. There exists a left approximate parametrix for the operator $P$ in (1.1), i.e. there exists a symbol $q(x, \xi, \tau, \lambda)$ such that, for $N \in \mathbb{N}$, a large integer,

a) $q(x, \xi, \tau, \lambda)=\sum_{j=1}^{3} q_{j}(x, \xi, \tau, \lambda)$ with

1. $q_{1}=\tau^{\frac{2 k}{q}-\frac{2}{q}} \lambda^{\frac{2 k}{q}+\frac{2(q-1)}{q}} \sum_{s} \tilde{c}_{s}(\tau \lambda)^{-\frac{2 k}{q} s} \sigma\left(E E^{*}\right)$ where $s<N+1+\frac{q-1}{k}$ and $\sigma\left(E E^{*}\right) \in \mathscr{H}_{q}^{0}$;

2. $q_{2}=\tau^{\frac{2 k}{q}-\frac{2}{q}} \lambda^{\frac{2 k}{q}+\frac{2(q-1)}{q}} \sum \tilde{c}_{s}(\tau \lambda)^{-\frac{2 k}{q} s} \sigma\left(E E^{*} P_{k}\left(1+F_{0} P_{k}\right)^{-1} F_{0}\right)$ where $\sigma\left(E E^{*} P_{k}\left(1+F_{0} P_{k}\right)^{-1} F_{0}\right) \in \mathscr{H}_{q}^{-\frac{2 k}{q}}$

3. $q_{3}=\sigma\left(\left(1+F_{0} P_{k}\right)^{-1} F_{0}\right)$ belongs to $S_{q}^{0,-2}$.

b) we have

$$
\begin{aligned}
& (Q \circ P) u(x, t)=u(x, t)+ \\
& +\left(\frac{\lambda}{2 \pi}\right)^{2} \iint e^{i \lambda\left(x-x^{\prime}\right) \eta+i \lambda\left(t-t^{\prime}\right) \tau} a(x, \xi, \tau, \lambda) u\left(x^{\prime}, t^{\prime}\right) d x^{\prime} d t^{\prime} d \xi d \tau
\end{aligned}
$$

modulo terms in OP $\mathscr{H}_{q}^{-\frac{2 k}{q} N-\frac{1}{q}}$, where $a=\tau^{-\frac{2 k}{q} N} \lambda^{-\frac{2 k}{q} N} \sigma\left(E E^{*}\right)$.

\section{The local a priori estimate via FBI}

We recall some basic notions related to the Fourier-Bros-Iagolnitzer transformation, fore more details see [10]. We consider the F.B.I.-transformation with the classical phase function

$$
T u(z, \lambda)=\int_{\mathbb{R}^{2}} e^{-\frac{\lambda}{2}(z-y)^{2}} u(y) d y
$$

where $\lambda \geq 1, z=\left(z_{1}, z_{2}\right) \in \mathbb{C}^{2}$ and $y=(x, t) \in \mathbb{R}^{2}$. Let

$$
\varphi_{0}(z)=\sup _{y \in \mathbb{R}^{2}}\left(-\operatorname{Im}\left(\frac{i}{2}(z-y)^{2}\right)\right)=\frac{\left(\operatorname{Im} z_{1}\right)^{2}}{2}+\frac{\left(\operatorname{Im} z_{2}\right)^{2}}{2}
$$

be the plurisubharmonic weight function associated to the classical phase function. We put

$$
\varphi_{0,1}\left(z_{1}\right)=\frac{\left(\operatorname{Im} z_{1}\right)^{2}}{2} \quad \text { and } \quad \varphi_{0,2}\left(z_{2}\right)=\frac{\left(\operatorname{Im} z_{2}\right)^{2}}{2} .
$$

We also recall that $T$ is associated to a canonical transformation $\mathcal{H}_{T}$ from $\mathbb{C}^{4}$ in itself:

$$
(w, i(z-w)) \longmapsto(z, i(z-w))
$$


such that

$$
\mathcal{H}_{T}\left(T^{*} \mathbb{R}^{2}\right)=\Lambda_{\varphi_{0}}=\left\{\left(z,-2 i \partial_{z} \varphi_{0}(z)\right) \mid z \in \mathbb{C}^{2}\right\},
$$

$\mathcal{H}_{T}(x, t, \xi, \tau)=(x-i \xi, t-i \tau, \xi, \tau) . \Lambda_{\varphi_{0}}$ is an I-Lagrangian, $\mathbb{R}$-symplectic (totally real) subspace in $\mathbb{C}^{4}$. If $u \in \mathscr{S}^{\prime}\left(\mathbb{R}^{2}\right)$ then $T u$ is an holomorphic function of $z \in \mathbb{C}^{2}$ such that $|T u(z, \lambda)| \leq \mathcal{O}_{\lambda}(1)\langle z\rangle^{N} e^{\lambda \varphi_{0}(z)}$ for some $N$ depending on $u$ where $\langle z\rangle=\left(1+|z|^{2}\right)^{1 / 2}$ and $\mathcal{O}_{\lambda}(1)$ denotes a uniformly bounded quantity when $\lambda \rightarrow+\infty$; moreover if $u$ belongs to $L^{2}\left(\mathbb{R}^{2}\right)$ then $T u \in L^{2}\left(\mathbb{C}^{2} ; e^{-\lambda \varphi_{0}(z)} L(d z)\right)$ where $L(d z)$ is the Lebesgue measure in $\mathbb{R}^{4}$, $L(d z)= \pm(2 i)^{-2} d z \wedge d \bar{z}$.

We recall briefly the characterization of analytic and $C^{\infty}$ wave front set in the F.B.I. setting (see Sjöstrand [10]): a point $\left(y_{0}, \eta_{0}\right) \in \mathbb{R}^{4}$ does not belong to $W F_{a}(u)$ if and only if there exist a positive constant $\varepsilon$, a neighborhood $\Omega$ of $y_{0}-i \eta_{0}$ in $\mathbb{C}^{2}$ and a positive constant $C_{\Omega}$, depending on $\Omega$, such that

$$
|T u(z, \lambda)| e^{-\frac{\lambda}{2} \varphi_{0}(z)} \leq C_{\Omega} e^{-\varepsilon \lambda} \quad \forall z \in \Omega ;
$$

analogously we say that $\left(y_{0}, \eta_{0}\right) \notin W F(u)$ if there exists a neighborhood $\Omega$ of $y_{0}-i \eta_{0}$ in $\mathbb{C}^{2}$ such that

$$
|T u(z, \lambda)| e^{-\frac{\lambda}{2} \varphi_{0}(z)} \leq C_{\Omega} \lambda^{-N} \quad \forall N \in \mathbb{N} \text { and } \forall z \in \Omega .
$$

A direct computation gives $T\left(D_{x} u\right)=D_{z_{1}} T u, T\left(D_{t} u\right)=D_{z_{2}} T u$ and $T(x u)=\left(z_{1}+i \lambda^{-1} D_{z_{1}}\right) T u$; it is not more difficult to compute $T P$ directly.

We denote by $P$ the zero order Kohn operator, $\lambda^{-2} P$, after the F.B.I. and we put $\tilde{\Sigma}=\mathcal{H}_{T}(\Sigma)=\Sigma^{\mathbb{C}} \cap \Lambda_{\varphi_{0}}$, where $\Sigma$ is the characteristic set of $P$ before the F.B.I. transformation and $\Sigma^{\mathbb{C}}$ denotes the complexification of $\Sigma$.

In order to study the wave front set we need an a priori estimate on the F.B.I. side. This estimate is obtained by a technique inspired by [11]. We have an additional difficulty do to the fact that we need to work with Hermite operators on the F.B.I. side.

Let $\left(z_{0}, \zeta_{0}\right) \in \mathcal{H}_{T}(\Sigma)$ and $W$ a neighborhood of $\left(z_{0}, \zeta_{0}\right)$ such that $W \cap \Lambda_{\varphi_{0}}$ is a suitably small neighborhood of $\left(z_{0}, \zeta_{0}\right)$ in $\Lambda_{\varphi_{0}}$. Let $F$ be a $C^{\omega}$ map

$$
F: W \longrightarrow \mathbb{C}^{4}
$$

such that

1. $F$ is close to the identity map in the $C^{1}$ norm, $\|F-I\|_{C^{1}}=\mathcal{O}(\varepsilon)$, where $\varepsilon$ is a small positive parameter; we want that $F\left(W \cap \Lambda_{\varphi_{0}}\right)$ has a injective projection onto $\mathbb{C}_{z}^{2}$. Thus it is a graph.

2. There exists a real valued non negative plush function $\varphi$ such that $\varphi(z)=\varphi_{1}\left(z_{1}\right)+\varphi_{2}\left(z_{2}\right)$ and

$$
F\left(W \cap \Lambda_{\varphi_{0}}\right)=\Lambda_{\varphi} \cap F(W)
$$

where $\Lambda_{\varphi}=\left\{\left(z,-2 i \partial_{z} \varphi(z)\right) \mid z \in \mathbb{C}^{2}\right\}$. 
Moreover we put $\varphi\left(z_{0}\right)=\varphi_{0}\left(z_{0}\right)$. Then $\varphi$ is as close as we want to $\varphi_{0}$ if $F$ is close to the identity (in a suitable small neighborhood of $\left(z_{0}, \zeta_{0}\right)$.)

We remark that $\Lambda_{\varphi}$ is in a small tubular neighborhood of $\Lambda_{\varphi_{0}}$.

We define $L_{\varphi}^{2}(\Omega)$ as the set of all locally square integrable functions defined on $\Omega$ equipped with the norm

$$
\|u\|_{\varphi, \Omega}^{2}=\int_{\Omega}|u(z)|^{2} e^{-2 \lambda \varphi(z)} L(d z)
$$

and $L_{\varphi, \Omega}^{2,2}$ as the set of all locally square integrable functions defined on $\Omega$ equipped with the norm

$$
\|\| u \|_{\varphi, \Omega}^{2}=\int_{\Omega}|u(z)|^{2}\left(d^{2}(z)+\lambda^{-2 \frac{q-1}{q}}\right)^{2} e^{-2 \lambda \varphi(z)} L(d z),
$$

where $d=d_{\Sigma \mathbb{C} \cap \Lambda_{\varphi}}$ is $\left(d \circ \mathcal{H}_{T}\right)_{\Lambda_{\varphi}}$ (d, in the last formula, is the distance function defined in the previous section.) Since $F$ is close to the identity we have that $p_{\left.2\right|_{\Lambda_{\varphi}}} \in S_{q}^{0,2}$ and $p_{\left.k\right|_{\Lambda_{\varphi}}} \in S_{q}^{0,2+\frac{2 k}{q-1}}$, i.e.

$$
\left|p_{\left.2\right|_{\Lambda_{\varphi}}}\right| \lesssim\left(d^{2}+\lambda^{-2(q-1) / q}\right) \quad \text { and } \quad\left|p_{\left.k\right|_{\Lambda_{\varphi}}}\right| \lesssim\left(d^{2}+\lambda^{-2(q-1) / q}\right)^{\frac{k}{q-1}+1} \text {. }
$$

Let $\left(z^{0}, \zeta^{0}\right)=(0,-i, 0,1)=\mathcal{H}_{T}(\rho)$, where $\rho=(0,0,0,1) \in \Sigma^{+}$, let $\left(z^{1}, \zeta^{1}\right)=F\left(z^{0}, \zeta^{0}\right) \in \Sigma^{\mathbb{C}} \cap \Lambda_{\varphi}$, and let $\Omega, \Omega_{1}$ be open suitably small neighborhoods of $z^{0}\left(=z^{1}\right)$ in $\mathbb{C}^{2}$ with $\Omega_{1} \subset \subset \Omega$. The open set $\Omega$ is strictly contained in $\prod_{\mathbb{C}_{z}^{2}}(W)$, where $\prod_{\mathbb{C}_{z}^{2}}$ is the projection on $\mathbb{C}_{z}^{2}$ and $W$ is the domain of $F$ in $\mathbb{C}_{z, \zeta}^{4}$.

For an analytic symbol $q(z, \zeta, \lambda)$ we define the corresponding pseudodifferential operator acting on holomorphic functions as

$$
Q u(z)=\left(\frac{\lambda}{2 \pi}\right)^{2} \iint e^{i \lambda(z-w) \zeta} q((z+w) / 2, \zeta, \lambda) u(w) d w \wedge d \zeta .
$$

The integral is taken along an integration contour of the form

$$
\Gamma:=\zeta=\frac{2}{i} \frac{\partial \varphi}{\partial z}\left(\frac{z+w}{2}\right)+i C \overline{(z-w)} \quad \text { with }|z-w| \leq r
$$

where $\varphi$ is the phase function given above, $r$ is a small positive constant such that $\operatorname{dist}\left(\Omega_{1} ; \complement \Omega\right)>r$ and $C$ is a positive suitable constant such that

$$
\left|e^{i \lambda(z-w) \zeta}\right| e^{-\lambda \varphi(z)} e^{\lambda \varphi(w)} \leq e^{-\delta|z-w|^{2}}
$$

for some $\delta>0$. 
Since we need to work with Hermite operators on the F.B.I. side, we must introduce an adapted integration path for this type of operators, taking into account the anisotropic behavior of the operator $P$ w.r.t. the $x$ variable. We introduce the integration contour

$$
\left\{\begin{array}{l}
\zeta_{1}=\frac{2}{i} \frac{\partial \varphi_{1}}{\partial z}\left(\frac{z_{1}+w_{1}}{2}\right)+i C\left(\left(z_{1}^{\prime}-w_{1}^{\prime}\right)^{q-1}-i\left(z_{1}^{\prime \prime}-w_{1}^{\prime \prime}\right)^{\frac{1}{q-1}}\right) \\
\zeta_{2}=\frac{2}{i} \frac{\partial \varphi_{2}}{\partial z}\left(\frac{z_{2}+w_{2}}{2}\right)+i C \overline{\left(z_{2}-w_{2}\right)}
\end{array}\right.
$$

where $z_{j}=z_{j}^{\prime}+i z_{j}^{\prime \prime}$, where $z_{j}^{\prime}, z_{j}^{\prime \prime}$ are real, and $C$ is a suitably small constant. We remark, that, in the case $q=2$, the above integration path is the usual one and we only need to chose $C$ suitably small.

Denote by $\tilde{Q}$ the approximate parametrix of $P$ after the F.B.I. transformation

$$
\tilde{Q} u(z)=\left(\frac{\lambda}{2 \pi}\right)^{2} \sum_{j=1}^{3} \iint_{\Gamma_{j}} e^{i \lambda(z-w) \zeta} \tilde{q}_{j}\left(\left(z_{1}+w_{1}\right) / 2, \zeta, \lambda\right) u(w) d w \wedge d \zeta
$$

where $\tilde{q}_{j} \circ \mathcal{H}_{T}=q_{j}$ and the integration contour $\Gamma_{j}$ is of the form (3.4) for $j=1,2$ and of the form (3.3) for $j=3$.

Let $\Omega_{2}$ be an open neighborhood of $z_{0}$ such that

$$
\Omega_{2} \subset \subset \Omega_{1} \subset \subset \Omega \text { and } \operatorname{dist}\left(\Omega_{2}, \subset \Omega_{1}\right)>r .
$$

Our purpose is to obtain an estimate of the form

$$
\|u\|_{\varphi, \Omega_{2}} \lesssim \lambda^{\frac{2 k}{q}+\frac{3(q-1)}{q}-\frac{1}{q}}\|P u\|_{\varphi, \Omega_{1}}+\|\| u \|_{\varphi, \Omega \backslash \Omega_{2}} .
$$

Let $v$ be an holomorphic function on $\Omega$, we want to show that

$$
\|\tilde{Q} v\|_{\varphi, \Omega_{2}} \lesssim \lambda^{\frac{2 k}{q}+\frac{3(q-1)}{q}-\frac{1}{q}}\|v\|_{\varphi, \Omega_{1}}, .
$$

We recall that if $K$ is an operator defined by an integral kernel $k$ :

$$
K u(z)=\int_{O} k(z, w) u(w) L(d w)
$$

where $O$ is a suitable complex domain, we have

$$
\begin{aligned}
& \|\| K u\left\|_{\varphi, \Omega}^{2}=\right\|\left(d^{2}(\cdot)+\lambda^{-2 \frac{q-1}{q}}\right) K u \|_{\varphi, \Omega}^{2} \leq \\
& \quad \leq\|u\|_{\varphi, O}^{2} \iint_{\Omega \times O} e^{-2 \lambda(\varphi(z)-\varphi(w))}\left|\left(d^{2}(z)+\lambda^{-2 \frac{q-1}{q}}\right) k(z, w)\right|^{2} L(d z) L(d w) .
\end{aligned}
$$


In order to obtain (3.5) we need to estimate kernels of the form

$$
e^{-\lambda(\varphi(z)-\varphi(w))}\left(d^{2}(z)+\lambda^{-2(q-1) / q}\right) k(z, w) .
$$

We start with $\tilde{Q}_{3}$ and we want to show that

$$
\left\|\tilde{Q}_{3} v\right\|_{\varphi, \Omega_{2}} \lesssim\|v\|_{\varphi, \Omega_{1}} .
$$

In this situation we work with the classical integration contour. Its kernel $k$ has the form

$$
e^{i \lambda(z-w) \zeta} \tilde{q}_{3}\left(\frac{z_{1}+w_{1}}{2}, \zeta, \lambda\right) \frac{\partial \zeta}{\partial \bar{w}} .
$$

We have that $\left|\partial_{\bar{w}} \zeta\right| \leq$ const. By the Taylor expansion we can replace $\tilde{q}_{3}$ in the above formula with

$$
\begin{aligned}
\tilde{q}_{3}\left(z_{1}, \frac{2}{i} \frac{\partial \varphi}{\partial z}(z), \lambda\right)+\frac{\partial \tilde{q}_{3}}{\partial \zeta_{1}}\left(z_{1}, \frac{2}{i} \frac{\partial \varphi}{\partial z}, \lambda\right)\left(\zeta_{1}-\frac{2}{i} \frac{\partial \varphi_{1}}{\partial z_{1}}(z)\right)+ \\
+\frac{\partial \tilde{q}_{3}}{\partial \zeta_{2}}\left(z_{1}, \frac{2}{i} \frac{\partial \varphi}{\partial z}(z), \lambda\right)\left(\zeta_{2}-\frac{2}{i} \frac{\partial \varphi_{2}}{\partial z_{2}}(z)\right)+\frac{\partial \tilde{q}_{3}}{\partial z_{1}}\left(z_{1}, \frac{2}{i} \frac{\partial \varphi}{\partial z}(z), \lambda\right)\left(\frac{z_{1}-w_{1}}{2}\right)+ \\
+\mathcal{R}_{1, \lambda}\left(|z-w|^{2}\right)+\mathcal{R}_{2, \lambda}\left(\left|\zeta-\frac{2}{i} \frac{\partial \varphi}{\partial z}(z)\right|^{2}\right)
\end{aligned}
$$

where $R_{1, \lambda}, R_{2, \lambda}$ denote holomorphic symbols which are uniformly $\mathcal{O}\left(\lambda^{\frac{2}{q}-1}\right)$. Since $F$ is close to identity and the Lipschitz norm of $F-I$ is bounded by $\mathcal{O}(\varepsilon)$ we have $\nabla \varphi(z+h)-\nabla \varphi(z)=\left[\nabla^{2} \varphi_{0}+\mathcal{O}(\varepsilon)\right](z-h)$; hence $\left|\zeta_{j}+2 i \partial_{z_{j}} \varphi_{j}(z)\right| \lesssim\left|z_{j}-w_{j}\right|$ for $j=1,2$. We have that $\tilde{q}_{\left.3\right|_{\Lambda \varphi}}$ is in the class $S_{q}^{0,-2}$ with respect to the distance function defined above; so we conclude that $\left|\tilde{q}_{\left.3\right|_{\Lambda_{\varphi}}}\right| \lesssim\left(d^{2}+\lambda^{-2(q-1) / q}\right)^{-1}$. In view of the canonical transformation associated to the F.B.I. transformation we have that

$$
\left|\partial_{\zeta_{1}} \tilde{q}_{\left.3\right|_{\Lambda_{\varphi}}}\right| \lesssim \lambda^{\frac{1}{q}}\left(d^{2}+\lambda^{-2(q-1) / q}\right)^{-1}
$$

On the other hand we have

$\left|\partial_{z_{1}} \tilde{q}_{\left.3\right|_{\Lambda_{\varphi}}}\right| \lesssim \lambda^{\frac{1}{q}}\left(d^{2}+\lambda^{-2(q-1) / q}\right)^{-1} \quad$ and $\quad\left|\partial_{\zeta_{2}} \tilde{q}_{\left.3\right|_{\Lambda_{\varphi}}}\right| \lesssim \lambda^{-1}\left(d^{2}+\lambda^{-2(q-1) / q}\right)^{-1}$.

The remainder terms give rise to an operator that is uniformly $\mathcal{O}\left(\lambda^{\frac{2}{q}-1}\right)$ acting from $L_{\varphi, \Omega_{2}}^{2,2}$ to $L_{\varphi, \Omega_{1}}^{2}$. We can estimate $\left\|\tilde{Q}_{3} v\right\|_{\varphi, \Omega_{2}}$ with

$$
\begin{array}{r}
\|v\|_{\varphi, \Omega_{1}}\left(\frac{\lambda}{2 \pi}\right)^{2} \iint e^{-\delta \lambda|z-w|^{2}}\left(1+\frac{1}{\lambda}\left|z_{2}-w_{2}\right|+\lambda^{\frac{1}{q}} \mid z_{1}-\right. \\
\left.-w_{1} \mid\right)^{2} L\left(d z^{\prime}\right) L(d z) \\
+\mathcal{O}\left(\lambda^{\frac{2}{q}-1}\right)\|v\|_{\varphi, \Omega_{1}} .
\end{array}
$$

Since $q$ is an even integer and in this way $\frac{1}{q}-\frac{1}{2}$ is smaller or equal to zero. 
Next we want to estimate $\left\|\tilde{Q}_{1} v\right\|_{\varphi, \Omega_{2}}$ where $\tilde{Q}_{1}$ is realized on the integration path (3.4). Its kernel is

$$
\begin{aligned}
e^{i \lambda(z-w) \zeta} e^{-\frac{\lambda \zeta_{2}}{q}\left(z_{1}+i \zeta_{1}\right)^{q}-i \lambda\left(z_{1}+i \zeta_{1}\right) \zeta_{1}} \sum_{s} \tilde{c}_{s}\left(\zeta_{2} \lambda\right)^{-\frac{2 k}{q} s} \times & \\
& \times \int_{\mathbb{R}} e^{-i \lambda y \zeta_{1}} e^{-\frac{\lambda \zeta_{2}}{q} y^{q}} d y \frac{\partial \zeta_{1}}{\partial \bar{w}_{1}} \frac{\partial \zeta_{2}}{\partial \bar{w}_{2}}
\end{aligned}
$$

where $s<N+1+\frac{q-1}{k}$. We have that

$$
\left|\frac{\partial \zeta_{1}}{\partial \bar{w}_{1}}\right| \leq C_{1}\left(1+\left(z_{1}^{\prime}-w_{1}^{\prime}\right)^{q-2}+\left(z_{1}^{\prime \prime}-w_{1}^{\prime \prime}\right)^{\frac{1}{q-1}-1}\right)
$$

and $\left|\partial_{\bar{w}_{2}} \zeta_{2}\right| \leq$ cost. On $\Gamma$, using the same technique of [4] and [5], we have

$$
\begin{array}{r}
\left|e^{i \lambda(z-w) \zeta-\lambda(\varphi(z)-\varphi(w))} e^{-\frac{\lambda \zeta_{2}}{q}\left(z_{1}+i \zeta_{1}\right)^{q}-i \lambda\left(z_{1}+i \zeta_{1}\right) \zeta_{1}} \int_{\mathbb{R}} e^{-i \lambda y \zeta_{1}} e^{-\frac{\lambda \zeta_{2}}{q} y^{q}} d y\right| \lesssim \\
\lesssim e^{-\lambda \delta_{2}\left|z_{2}-w_{2}\right|^{2}} e^{-\lambda \varepsilon_{1}\left|z_{1}^{\prime}-w_{1}^{\prime}\right|^{q}-\lambda \varepsilon_{2}\left|z_{1}^{\prime \prime}-w_{1}^{\prime \prime}\right|^{\frac{q}{q-1}}}
\end{array}
$$

where $\varepsilon_{1}$ and $\varepsilon_{2}$ are suitable constants depending on the constant $C$ in (3.4) and $q$. We can conclude that

$$
\left\|\tilde{Q}_{1} v \mid\right\|_{\varphi, \Omega_{2}} \lesssim \lambda^{\frac{2 k}{q}+\frac{3(q-1)}{q}-\frac{1}{q}}\|v\|_{\varphi, \Omega_{1}} .
$$

It only remains to estimate $\tilde{Q}_{2}$. We realize it with the integration path (3.4). Its kernel is

$$
\begin{aligned}
e^{i \lambda(z-w) \zeta} e^{-\frac{\lambda \zeta_{2}}{q}\left(z_{1}+i \zeta_{1}\right)^{q}-i \lambda\left(z_{1}+i \zeta_{1}\right) \zeta_{1}} \sum_{s} & \tilde{c}_{s}\left(\zeta_{2} \lambda\right)^{-\frac{2 k}{q} s} \times \\
& \times \int_{\mathbb{R}} e^{-i \lambda y \zeta_{1}} p\left(y, \zeta_{2}\right) e^{-\frac{\lambda \zeta_{2}}{q} y^{q}} d y \frac{\partial \zeta_{1}}{\partial \bar{w}_{1}} \frac{\partial \zeta_{2}}{\partial \bar{w}_{2}} .
\end{aligned}
$$

where $p\left(y, \zeta_{2}\right)$ is a polynomial of order $2 k$ w.r.t. the variable $y$. Actually $p_{2}\left(y, \zeta_{2}\right) \sim y^{2 k} \tilde{p}_{2}\left(y, \zeta_{2}\right)$. On the integral contour $\Gamma$, using the same technique of [4] and [5], we have

$$
\begin{array}{r}
\left|e^{i \lambda(z-w) \zeta-\lambda(\varphi(z)-\varphi(w))} e^{-\frac{\lambda \zeta_{2}}{q}\left(z_{1}+i \zeta_{1}\right)^{q}-i \lambda\left(z_{1}+i \zeta_{1}\right) \zeta_{1}} \int_{\mathbb{R}} e^{-i \lambda y \zeta_{1}} p\left(y, \zeta_{2}\right) e^{-\frac{\lambda \zeta_{2}}{q} y^{q}} d y\right| \lesssim \\
\lesssim \lambda^{-\frac{2 k}{q}} e^{-\lambda \delta_{2}\left|z_{2}-w_{2}\right|^{2}} e^{-\lambda \varepsilon_{1}\left|z_{1}^{\prime}-w_{1}^{\prime}\right|^{q}-\lambda \varepsilon_{2}\left|z_{1}^{\prime \prime}-w_{1}^{\prime \prime}\right|^{\frac{q}{q-1}}}
\end{array}
$$

We can conclude that

$$
\left\|\tilde{Q}_{2} v\right\|\left\|_{\varphi, \Omega_{2}} \lesssim \lambda^{\frac{3(q-1)}{q}-\frac{1}{q}}\right\| v \|_{\varphi, \Omega_{1}} .
$$

Then (3.5) follows. 
We replace $v$ by $P u$ in (3.5) and we obtain

$$
\lambda^{\frac{2 k}{q}+\frac{3(q-1)}{q}-\frac{1}{q}}\|P u\|_{\varphi, \Omega_{1}} \gtrsim\|\tilde{Q} P u\|_{\varphi, \Omega_{2}} \gtrsim\|\| u\left\|_{\varphi, \Omega_{2}}-\right\| \tilde{Q} P u-u \|_{\varphi, \Omega_{2}} .
$$

Then

$$
\|\| u\left\|_{\varphi, \Omega_{2}} \lesssim \lambda^{\frac{2 k}{q}+\frac{3(q-1)}{q}-\frac{1}{q}}\right\| P u\left\|_{\varphi, \Omega_{1}}+\right\| \tilde{Q} P u-u \|_{\varphi, \Omega_{2}} ;
$$

Since the Weyl composition and the linear canonical transformation commute, using (2.12), we have

$$
\tilde{Q} \# P=1+\tilde{A} \quad \bmod \left(\lambda^{-\frac{2 k}{q} N-\frac{1}{q}}\right) .
$$

The second term in the r. h. s. of (3.6) can be estimated as follows

$$
\|\tilde{Q} P u-u \mid\|_{\varphi, \Omega_{2}} \lesssim\|L u\|_{\varphi, \Omega_{2}}+\|\tilde{A} u\|_{\varphi, \Omega_{2}},
$$

where, for a holomorphic function $v$ defined in $\Omega$, we set

$$
L v(z)=v(z)-\left(\frac{\lambda}{2 \pi}\right)^{2} \iint e^{i \lambda(z-w) \zeta} v(w) d w d \zeta
$$

the integral is performed along the contour $\zeta=-2 i \partial_{z} \varphi(z)-i C \overline{(z-w)}$. Let us estimate now the first term in the r. h. s. of (3.7). We recall that

$$
\|L u\|_{\varphi, \Omega_{2}} \lesssim e^{-\lambda / C}\|u\|_{\varphi, \Omega} .
$$

(See Sjöstrand [11] and [10].)

Using the Stokes theorem and the deformation argument

$$
-2 i \partial_{z} \varphi\left(z t+(1-t) z^{\prime}\right)-i C \overline{(z-w)},
$$

with $t \in\left[\frac{1}{2}, 1\right]$, we can replace the above integration path

$$
\left(-2 i \partial_{z} \varphi(z)-i C \overline{(z-w)}\right)
$$

with the contour

$$
\zeta=-2 i \partial_{z} \varphi\left(\frac{z+z^{\prime}}{2}\right)-i C \overline{(z-w)}
$$

The operator $\tilde{A}$ is realized on the integration path (3.4) and its symbol is $\lambda^{-2 k N / q} \zeta_{2} \sigma\left(E E^{*}\right)(z+i \zeta, \zeta)$. The same technique used to estimate $\tilde{Q}_{1}$ allows us to estimate the second term in (3.7):

$$
\mid\|\tilde{A} u\|_{\varphi, \Omega_{2}} \lesssim \lambda^{-\frac{2 k}{q} N+\frac{3(q-1)}{q}-\frac{1}{q}}\|\| u \|_{\varphi, \Omega} .
$$


We choose $N$ such that

$$
2 k N+4>3 q
$$

e.g. $2 N=3 q$. Then (3.6) can be rewritten as

$$
\|u\|\left\|_{\varphi, \Omega_{2}} \lesssim \lambda^{\frac{2 k}{q}+\frac{3(q-1)}{q}-\frac{1}{q}}\right\| P u\left\|_{\varphi, \Omega_{1}}+\left(\frac{1}{\lambda^{\alpha}}+e^{-\lambda / C^{\prime}}\right)\right\| u \|_{\varphi, \Omega}
$$

where $\alpha=-\frac{2 k}{q} N+\frac{3(q-1)}{q}-\frac{1}{q}$.

Let $\lambda_{0}$ be such that $\lambda_{0}^{-\alpha}+e^{-\lambda_{0} / C^{\prime}}<1$. Then for every $\lambda \geq \lambda_{0}$ we have the

Proposition 3.1. Let $P$ be as in (1.1). Then on the F.B.I. side we have the a priori estimate

$$
\|u\|_{\varphi, \Omega_{2}} \lesssim \lambda^{\frac{2 k}{q}+\frac{3(q-1)}{q}-\frac{1}{q}}\|P u\|_{\varphi, \Omega_{1}}+\|u\|_{\varphi, \Omega \backslash \Omega_{2}} .
$$

where $\varphi$ has been defined at the beginning of Section 2 .

\section{The construction of the phase function}

In the next section we use a deformation argument of Holmgren type due to J. Sjöstrand [11] and [10].

First we construct a weight function $\varphi$ by solving a Hamilton-Jacobi equation for small times.

Let $r: W \rightarrow \mathbb{C}$ be a $C^{\infty}$ function ( $W$ is a neighborhood of $\left(z_{0} ; \zeta_{0}\right)$ whose space projection contains $\Omega$.) Consider

$$
\left\{\begin{array}{l}
\frac{\partial \varphi}{\partial s}(s, x)=(\operatorname{Re} r)\left(x, \frac{2}{i} \frac{\partial \varphi}{\partial x}(s, x)\right) \\
\varphi(0, x)=\varphi_{0}(x)
\end{array}\right.
$$

for $0 \leq s \leq \varepsilon_{0}$.

The solution of the above problem is constructed using the standard Hamilton-Jacobi theory with respect to the symplectic form

$$
\operatorname{Im} \sigma=\operatorname{Im}(d \xi \wedge d x) .
$$

Actually, setting $\varphi_{s}(x)=\varphi(s, x)$, we have

$$
\Lambda_{\varphi_{s}}=\exp \left(s H_{\operatorname{Re} r}^{\operatorname{Im} \sigma}\right) \Lambda_{\varphi_{0}} .
$$

The map $\exp \left(s H_{\operatorname{Re} r}^{\operatorname{Im} \sigma}\right)$ is the function $F$ of the previous section. 
We recall that, if $r$ is a holomorphic function on $W$ then we have

$$
H_{\operatorname{Re} r}^{\operatorname{Im} \sigma}=\widehat{H_{i r}}
$$

where $H_{i r}$ is the usual complex standard Hamilton field of $i r$ and $\widehat{H_{i r}}$ denotes the real part of $H_{i r}$, i.e. the real field that gives the same result as $H_{i r}$ when acting on holomorphic functions.

If $r$ is holomorphic in $W$ and real valued on $\Lambda_{\varphi_{0}}$ the solution of the above Hamilton-Jacobi problem is obtained as the restriction to the positive $s$-axis of the solution of the complex equation

$$
\begin{cases}\frac{\partial \varphi}{\partial s}(s, x) & =r\left(x, \frac{2}{i} \frac{\partial \varphi}{\partial x}(s, x)\right) \\ \varphi(0, x) & =\varphi_{0}(x)\end{cases}
$$

for $|s|<\varepsilon_{0}$.

Since $\mathbb{R}^{4}$ and $\Lambda_{\varphi_{0}}$ are isomorphic it is easier to construct the function $r$ in $\mathbb{R}^{4}$ near the characteristic point $(0,0,0,1) \in$ Char $P$.

Let us choose

$$
r(x, t, \xi, \tau)=t^{2}+(\tau-1)^{2}+C\left(\frac{x^{q}}{q}+\xi^{2}\right),
$$

where $C$ is a positive constant that makes $r$ as positive as we desire outside $\Sigma$. We point out that

$$
H_{r}(\rho) \in T \Sigma, \quad \text { for } \rho \in \Sigma \text {. }
$$

Then, on $\Lambda_{\varphi_{0}}$,

$$
r\left(z, \frac{2}{i} \frac{\partial \varphi_{0}}{\partial z}(z)\right) \sim\left|z_{2}-z_{2,0}\right|^{2}+\left\|z_{1}\right\|^{2}
$$

for every $z \in \Pi_{\mathbb{C}_{z}^{2}}\left(W \cap \Lambda_{\varphi_{0}}\right)$. Here $z_{2,0}$ is equal to $-i$ and the norm $\|\cdot\|$ is defined by

$$
\left\|z_{1}\right\|^{2}=z_{1}^{\prime q}+z_{1}^{\prime \prime 2}
$$

We remark that

$$
\varphi_{s}(z)=\varphi_{0}(z)+r\left(z, \frac{2}{i} \frac{\partial \varphi}{\partial z}(0, z)\right) s+\mathscr{O}\left(s^{2}\right) .
$$




\section{Proof of Theorem 1.1}

We start with the assertion on the analytic wave front set. Denote by $\left(x_{0}, \xi_{0}\right)$ the point $(0,0,0,1) \in$ Char $P$.

Our purpose is to show that if $\left(x_{0}, \xi_{0}\right) \notin W F_{a}(P u)$ then $\left(x_{0}, \xi_{0}\right) \notin$ $W F_{a}(u)$. We recall estimate (3.8):

$$
\|u\|_{\varphi, \Omega_{2}} \leq C\left(\lambda^{\frac{2 k}{q}+\frac{3(q-1)}{q}-\frac{1}{q}}\|P u\|_{\varphi, \Omega_{1}}+\|u\|_{\varphi, \Omega \backslash \Omega_{2}}\right)
$$

where $\Omega_{2} \subset \subset \Omega_{1} \subset \subset \Omega \subset W$ and $z_{0} \in \Omega_{2}, z_{0}=x_{0}-i \xi_{0}$.

Since $u$ is a tempered distribution before the FBI transform, we have that

$$
\|u\|_{\varphi_{0}, \Omega} \leq C \lambda^{N_{0}}
$$

for a certain $N_{0} \in \mathbb{N}$.

Since $P u$ is real analytic at the real point $\left(x_{0}, \xi_{0}\right)$ before the FBI transform, we have that

$$
\|P u\|_{\varphi_{0}, \Omega_{3}} \leq C_{1} e^{-\lambda / C_{1}}
$$

for a positive constant $C_{1}$; here $\Omega_{3}$ is a suitable neighborhood of $z_{0}$. Recalling that

$$
\varphi_{s}(z)-\varphi_{0}(z) \sim s\left(\left|z_{2}-z_{2,0}\right|^{2}+\left\|z_{1}\right\|^{2}\right),
$$

we obtain

$$
\|P u\|_{\varphi_{s}, \Omega_{1}} \leq \tilde{C} e^{-\lambda / \tilde{C}}
$$

for a positive constant $\tilde{C}$.

Since, on $\Omega \backslash \Omega_{2}, r \geq \alpha>0$, we have

$$
\varphi_{\left.s\right|_{\Omega \backslash \Omega_{2}}} \geq \varphi_{0}+\alpha_{1} s, \quad \alpha_{1}>0,
$$

so then

$$
\|\| u\|\|_{\varphi_{t}, \Omega \backslash \Omega_{2}} \leq C e^{-\lambda / C_{s}^{\prime}}\|u\|_{\varphi_{0}, \Omega_{1}} \leq C_{s} e^{-\lambda / C_{s}} .
$$

Hence the a priori estimate (5.1) implies that

$$
\|u\|_{\varphi_{s}, \Omega_{2}} \leq C_{2} e^{-\lambda / C_{2}} .
$$

Let now $\Omega_{4}$ be a sufficiently small neighborhood of $z_{0}$ such that

$$
\varphi_{s}(z)<\varphi_{0}(z)+\frac{1}{C_{4}}
$$

on $\Omega_{4}$. Then

$$
\|u\|_{\varphi_{0}, \Omega_{4}} \leq C_{3} e^{-\lambda / C_{3}},
$$

which means that $u$ is real analytic at $\left(x_{0}, \xi_{0}\right)$ before the FBI transform. This proves the assertion. 
We point out that the regularity at the characteristic points in $\Sigma^{-}$is much easier and well known since the operator $P$ loses microlocally only one derivative (i.e. it is maximally hypoelliptic.)

Finally we remark that estimate (3.8) implies $C^{\infty}$ hypoellipticity as well. In fact the only action we must take is to replace the exponential decay of the (F.B.I. transform of) $u$ with a rapidly decrease decay, i.e. $P u=$ $\mathcal{O}\left(\lambda^{-\infty}\right)$ uniformly in a neighborhood of $z_{0} \in \Omega_{2}$. Using the same "canonical deformation" argument yield that the error term, i.e. the second term on the right hand side of (3.8) is exponentially decreasing. This ends the proof of Theorem 1.1.

\section{References}

[1] Boutet De Monvel, L.: Hypoelliptic operators with double characteristics and related pseudodifferential operators. Comm. Pure Appl. Math. 27 (1974), 585-639.

[2] Bove, A., Derridu, M., Kohn, J. J. and Tartakoff, D. S.: Sums of squares of complex vector fields and (analytic-) hypoellipticity. Math. Res. Lett. 13 (2006), no. 5-6, 683-701.

[3] Grušin, V. V.: On a class of hypoelliptic operators. Mat. Sb. 83 (125) (1970) no. 3, 456-473. Math. USSR Sb. 12 (1970) no. 3, 458-476.

[4] KAng, H.: On the Fourier transform of $e^{-\psi(x)}$. Studia Math. 98 (1991) no. $3,231-234$.

[5] Chung, J., Kim, D. And Kim, S. K.: Fourier transform of exponential functions and Legendre transform. Math. Res. Lett. 5 (1998), no. 5, 629-635.

[6] Kohn, J. J.: Hypoellipticity and loss of derivatives. Ann. of Math. (2) 162 (2005), 943-986.

[7] Kwon, K. H.: Concatenations applied to analytic hypoellipticity of operators with double characteristics. Trans. Amer. Math. Soc. 283 (1984), $753-763$.

[8] Kwon, K. H.: Hypoellipticity and local solvability of operators with double characteristics. Comm. Partial Differential Equations 10 (1985), 525-542.

[9] Sjöstrand, J.: Parametrices for pseudodifferential operators with multiple characteristics. Ark. Mat. 12 (1974), 85-130.

[10] Sjöstrand, J.: Singularités analytiques microlocales. In Astérisque 95, 1-166. Soc. Math. France, Paris, 1982.

[11] Sjöstrand, J.: Analytic wavefront sets and operators with multiple characteristics. Hokkaido Math. J. 12 (1983), 392-433.

[12] Tartakoff, D. S.: Local analytic hypoellipticity for $\square_{b}$ on nondegenerate Cauchy-Riemann manifolds. Proc. Natl. Acad. Sci. U.S.A. 75 (1978), 3027-3028. 
[13] TartakofF, D.S.: The local real analyticity of solutions to $\square_{b}$ and the $\bar{\partial}$-Neumann problem. Acta Math. 145 (1980), 177-204.

[14] Treves, F.: Concatenations of second-order evolution equations applied to local solvability and hypoellipticity. Comm. Pure Appl. Math. 26 (1973), 201-250.

[15] Treves, F.: Analytic hypo-ellipticity of a class of pseudodifferential operators with double characteristics and applications to the $\bar{\partial}$-Neumann problem. Comm. Partial Differential Equations 3 (1978), 475-642.

Recibido: 16 de octubre de 2009

Gregorio Chinni

Dipartimento di Matematica

Università di Bologna

Piazza di Porta San Donato 5

40127 Bologna, Italy

chinni@dm.unibo.it 